\title{
Computer-Aided Diagnosis in Hysteroscopic Imaging
}

\author{
M. S. Neofytou, V. Tanos, I. Constantinou, E. C. Kyriacou, M. S. Pattichis, and C. S. Pattichis
}

\begin{abstract}
The paper presents the development of a computeraided diagnostic (CAD) system for the early detection of endometrial cancer. The proposed CAD system supports reproducibility through texture feature standardization, standardized multifeature selection, and provides physicians with comparative distributions of the extracted texture features. The CAD system was validated using 516 regions of interest (ROIs) extracted from 52 subjects. The ROIs were equally distributed among normal and abnormal cases. To support reproducibility, the RGB images were first gamma corrected and then converted into HSV and YCrCb. From each channel of the gamma-corrected $\mathrm{YCrCb}$, HSV, and RGB color systems, we extracted the following texture features: 1) statistical features (SFs), 2) spatial gray-level dependence matrices (SGLDM), and 3) gray-level difference statistics (GLDS). The texture features were then used as inputs with support vector machines (SVMs) and the probabilistic neural network (PNN) classifiers. After accounting for multiple comparisons, texture features extracted from abnormal ROIs were found to be significantly different than texture features extracted from normal ROIs. Compared to texture features extracted from normal ROIs, abnormal ROIs were characterized by lower image intensity, while variance, entropy, and contrast gave higher values. In terms of ROI classification, the best results were achieved by using SF and GLDS features with an SVM classifier. For this combination, the proposed CAD system achieved an $81 \%$ correct classification rate.
\end{abstract}

Index Terms-Classification, computer-aided diagnostic (CAD), computer-aided hysteroscopy, endometrial cancer, endoscopy, hysteroscopy, texture features.

\section{INTRODUCTION}

A MONG the female population of the United States, endometrial cancer is the most frequent cancer associated with the genital system. In the United States in 2013 it was estimated that over 49560 new cases would have been diagnosed with gynecological cancer of the uterine corpus with an associated estimate of 8190 deaths [1]. Early and accurate diagnosis of the endometrial cancer is of paramount importance.

Hysteroscopy is considered to be the gold standard technique for the diagnosis of intrauterine pathology, once symptoms or sonographic evidence are suspicious for intrauterine neoplasia [2], [3].

Manuscript received December 17, 2013; revised May 3, 2014 and June 16, 2014; accepted June 20, 2014. Date of publication June 25, 2014; date of current version May 7, 2015.

M. S. Neofytou, I. Constantinou, and C. S. Pattichis are with the Department of Computer Science, University of Cyprus, Nicosia 1678, Cyprus (e-mail: mneoph@ucy.ac.cy; ikonst01@ @cs.ucy.ac.cy; pattichi@ucy.ac.cy).

V. Tanos is with the Aretaeio Medical Center, Nicosia 2024, Cyprus (e-mail: v.tanos@aretaeio.com).

E. C. Kyriacou is with the Department of Computer Science and Engineering, Frederick University, Limassol 3080, Cyprus (e-mail: e.kyriacou@ frederick. ac.cy).

M. S. Pattichis is with the Department of Electrical and Computer Engineering, University of New Mexico, Albuquerque, NM 87131 USA (e-mail: pattichis@ece.unm.edu).

Color versions of one or more of the figures in this paper are available online at http://ieeexplore.ieee.org.

Digital Object Identifier 10.1109/JBHI.2014.2332760
During the exam, the gynecologist guides the hysteroscope connected to a medical camera inside the uterine cavity via the cervical canal. New endoscopic systems provide excellent visualization and magnification of subtle lesions [4]. Post- or perimenopausal bleeding is the most frequent symptom demanding hysteroscopy and endometrial curettage. Endometrial precancerous changes, especially in atrophic endometrium such as in the menopausal state, are difficult to diagnose even during hysteroscopic evaluation.

Hysteroscopic video/images direct the surgeon according to his or her training, knowledge and experience, empirically to evaluate the suspected regions of interest (ROIs) and take biopsies. This subjective approach can be supported by a computeraided diagnostic (CAD) system, which eventually may increase diagnostic accuracy. An in situ, real-time, quantitative analysis of the endometrial video/images could assist the endoscopist to recognize and isolate abnormal ROIs with higher probability.

Our group has proposed a standardized protocol to be followed for texture feature analysis of endoscopic images during hysteroscopy [5]. It was shown that: 1) gamma corrected images gave significantly better approximations to the calibrated images; 2) there was no significant difference in texture features between the panoramic and close up views and between small angles of the telescope; and 3) significant texture differences were found between texture features extracted from normal versus abnormal ROIs. Fig. 1 illustrates normal and abnormal ROIs of hysteroscopic images of the endometrium. In general, normal ROIs have higher gray scale median, are more homogeneous, and have lower contrast when compared to the abnormal ROIs [5].

The objective of this study was to develop a CAD system able to differentiate between normal and abnormal endometrial tissue in the early stages of endometrial cancer based on color texture analysis in hysteroscopic imaging.

Preliminary studies were published by our group in [5]-[7] where the usefulness of color texture analysis in differentiating between normal and abnormal endometrium ROIs was demonstrated. However, in this paper an integrated system covering all steps of CAD processing (video capture, gamma correction, multiscale texture analysis, feature extraction and selection, and classification) are demonstrated. Classification of hysteroscopy images of the endometrium using texture and vessel descriptors was recently published by Vlachokosta et al. [8] showing very promising results. Sheraizin and Sheraizin [9] proposed a hardware calibration protocol, for contrast improvement of hysteroscopic imaging with very good results according to the experts. A method of hysteroscopy video summarization and browsing based on the Jeffrey divergence of the color histograms of the consecutive frames was proposed by Scharcanski et al. [10], [11]. It was shown that this adaptive method identified associated key frames with less false positives (i.e., not 


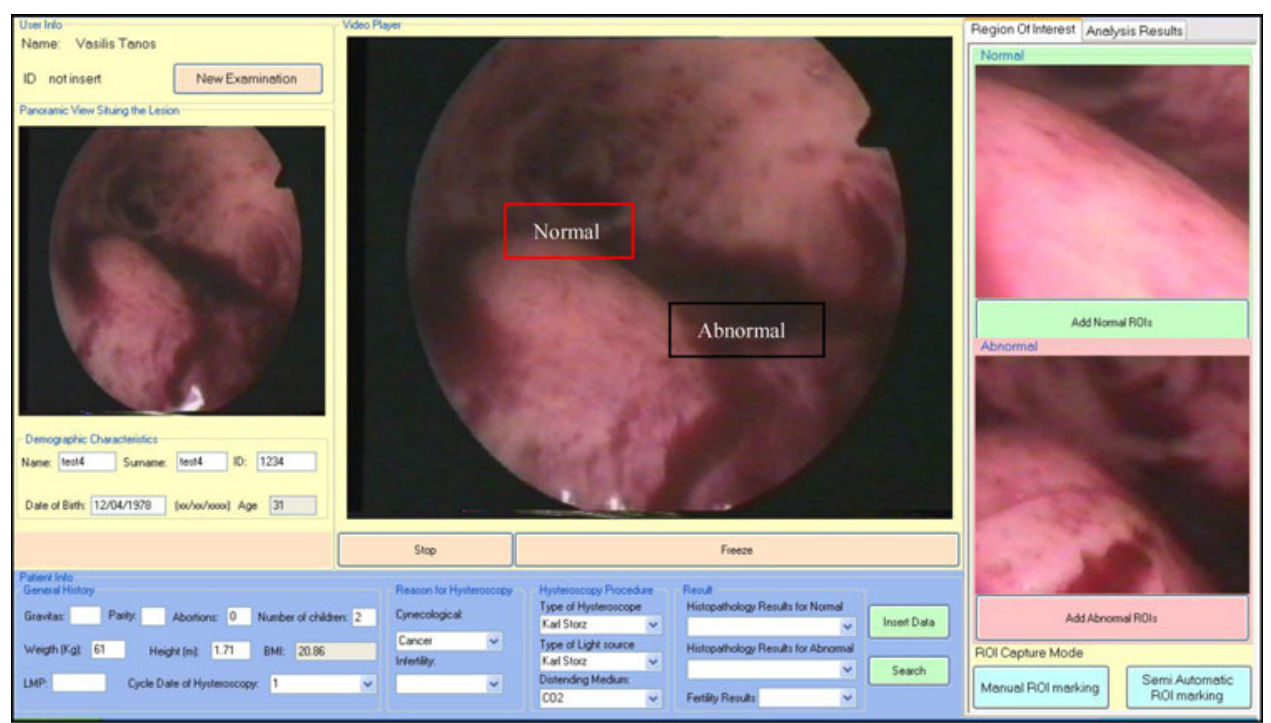

(a)

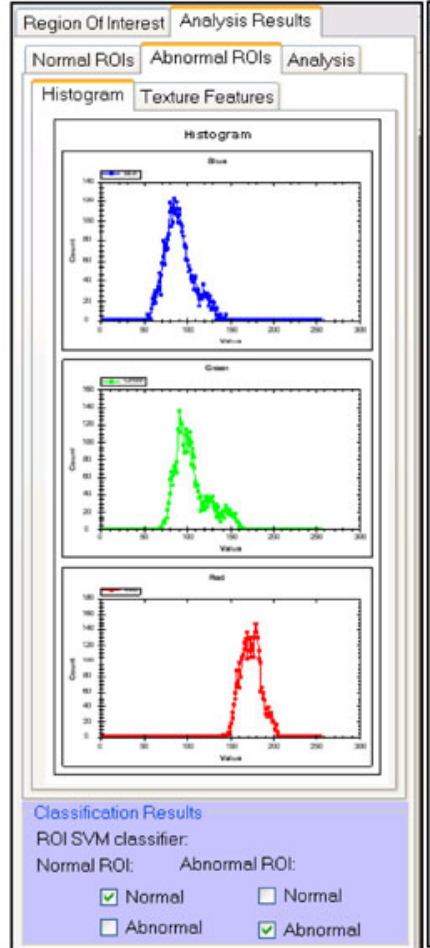

\begin{tabular}{|c|c|c|c|}
\hline \multicolumn{4}{|c|}{ Region Of Interest Analysis Results } \\
\hline \multicolumn{2}{|c|}{\begin{tabular}{|l|l|} 
Normal ROls & Abnormel ROls \\
\end{tabular}} & \multicolumn{2}{|l|}{ Analysis } \\
\hline \multicolumn{4}{|c|}{ Histogram Texture Features } \\
\hline & ROI1 & & $\hat{\imath}$ \\
\hline \multicolumn{4}{|l|}{ - SGLDM } \\
\hline Energy & 0.004 & & \\
\hline Contrast & 22.137 & & \\
\hline Correlation & 0.8403 & & \\
\hline Veriance & 69.306 & & \\
\hline Homogeneity & 0.3156 & & \\
\hline SumAverage & 255.088 & & \\
\hline SumVariance & 761.49 & & \\
\hline SumEntropy & 4.0728 & & \\
\hline Entropy & 6.0077 & & \\
\hline DiffVariance & 10.2107 & & \\
\hline Ditf Entropy & 2.1893 & & \\
\hline Corr Compl1 & -0.2487 & & \\
\hline Corr Compl2 & 0.8783 & & \\
\hline \multicolumn{4}{|l|}{ SF } \\
\hline Mean & 109.375 & & \\
\hline Veriance & $69.258 \mathrm{~s}$ & & \\
\hline Median & 110.504 & & \\
\hline Mode & 112 & & \\
\hline Skewness & -0.7373 & & \\
\hline Kurtosis & 3.1903 & & v \\
\hline$<$ & & $>$ & \\
\hline \multicolumn{4}{|l|}{ Classification Results } \\
\hline \multicolumn{4}{|c|}{ Abnormel ROt: } \\
\hline$\square$ Normal & & Normal & \\
\hline$\square$ Abnormal & & Abnormal & \\
\hline
\end{tabular}

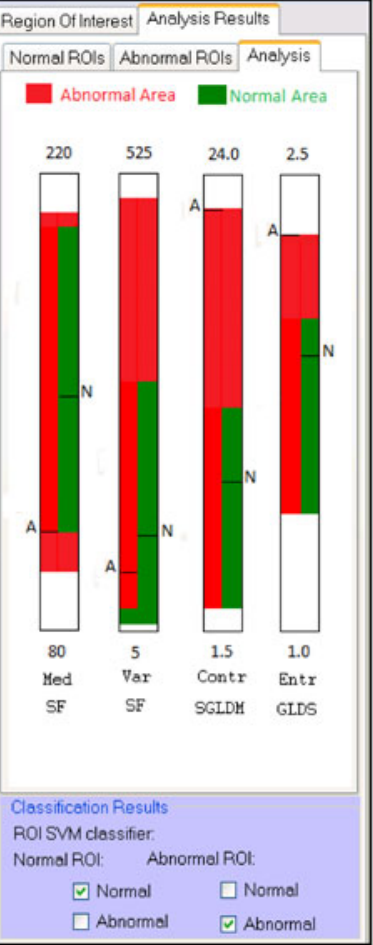

(b)

Fig. 1. An overview of the proposed hysteroscopy imaging CAD system consisting of: (a) Lower tab: patient information. Center column: video player with stop and freeze options (controlled by foot switch) and normal ROI shown in black border and abnormal ROI shown in white color line border. Right column: Regions of interest tab (showing normal and abnormal ROIs), analysis results tab, and ROI capture mode (manual or semi-automatic). (b) Left column: abnormal ROI RGB histograms. Middle column: texture features for the Y channel of the YCrCb system of the abnormal ROI. Right column: Mapping of the texture features SF median, SF variance, SGLDM contrast and GLDS entropy of the ROIs under investigation on bar plots. The lower and upper bounds of these plots are based on the analysis of normal and abnormal ROIs for the SF, SGLDM, and GLDS texture feature sets using the Y channel. The light gray column (green) of the feature map is used to illustrate the normal region, whereas the dark gray column (red) the abnormal region. It is shown that: (i) the normal ROI (N) investigated is within the normal bounds for the SF Med, SF variance, SGLDM contrast and GLDS entropy features; (ii) the abnormal ROI (A) investigated is within the normal bounds for the feature SF variance, but is out of the normal bounds (i.e. it falls in the abnormal regions) for the features SF median, SGLDM contrast, and GLDS entropy. The ROI classification results of the SVM classifier are shown on the lower part of the figure. It is illustrated that both the normal and abnormal ROIs under investigation were correctly classified.

clinically relevant segments, evaluated on 11 videos). Furthermore, a virtual hysteroscopy/laparoscopy system covering the aspects of collision detection, deformation computation, and path planning was proposed [12].
Compared to prior research, the fundamental innovations in the proposed research include:

1) Early cancer detection: The current study focuses on the detection of abnormal cases associated with occult cancer 
conditions at the earlier stages of initial cellular change to malignancy. This unique focus of our current study into the earlier cancer stages enables early detection and treatment prior to significant progression of the disease. In contrast, Vlachokosta et al. [8] had focused on later cancer stage cases that included the formation of neoangiogenesis and vessels structures that are associated with more advanced stages of carcinogenesis.

2) Reproducibility through standardization: Images extracted through hysteroscopy images can exhibit significant differences due to lighting variations and differences in viewing conditions. The current paper extends the standardized protocol reported in [5] for RGB images to $\mathrm{YCrCb}$ and $\mathrm{HSV}$ color spaces. The standardization process supports future integration of results from different clinics.

3) Standardized multifeature selection: Following standardization, we report texture features that exhibit significant differences between normal and abnormal ROIs based on the use of multicomparison methods.

4) CAD system advantages: The proposed system provides feedback to the physicians regarding the distribution of texture feature values associated with both normal and abnormal cases (see Fig. 1). Thus, our approach is to improve the standard practice of using visual inspection to determine regions for histology to a better informed approach that relies on statistical differences between standardized texture features from a clinically verified database.

The structure of this paper is as follows. Section II presents the methodology. Section III presents the results, and Section IV presents the discussion.

\section{Methodology}

In this section, the components of the proposed hysteroscopy imaging system are presented. A snapshot of the CAD system is demonstrated in Fig. 1. Moreover, a standardized protocol to support CAD in hysteroscopy (as well as in endoscopy and laparoscopy) image analysis is given in Fig. 2.

The original protocol described in [5] has been extended from covering RGB to include HSV and YCrCb. Furthermore, the new method includes ROI classification and reporting/diagnosis. The extensions from RGB to HSV and $\mathrm{YCrCb}$ allowed us to investigate more sophisticated color spaces that are associated with human perception [13]. In fact, as we shall describe in the Section III, the use of $\mathrm{YCrCb}$ gave the best classification results. Furthermore, the development of an ROI classification system provides the CAD system advantages detailed in Section I. Overall, the revised system includes the following components: 1) calibration and color correction estimation parameters; 2) video acquisition, ROI selection, and color correction; 3) texture analysis and classification (new); and 4) reporting/diagnosis (new).

\section{A. Dataset}

A total of 40 videos were recorded from 40 patients: 1) 18 patients with postmenopausal uterine bleedings and/or
Calibration and Color Correction Estimation Parameters:

1. Calibrate the camera following the guidelines by the manufacturer (i.e. white balance). This provides optimal-viewing calibration that will be environmentally dependable.

2. Capture the color palette ROIs and their corresponding digitally generated values based on the data given by the manufacturer.

3. Calculate the gamma correction parameters.

Video Acquisition, ROI Selection and Color Correction:

4. Acquire images at an angle that is nearly orthogonal to the object under investigation (only allowing 3 degree deviation) and at distances of $3 \mathrm{~cm}$ (close up) to $5 \mathrm{~cm}$ (panoramic).

5. ROIs selection:

a. Manually crop ROIs of $64 \times 64$ pixels of suspicious areas to be investigated and/or

b. Semi-automatically select a central region of $256 \times 256$ pixels, consisting of 16 ROIs of $64 \times 64$ pixels to be investigated.

6. ROIs are $\gamma$-corrected and visually assessed.

Texture Analysis and Classification (new)

7. Convert the ROIs from RGB to the $\mathrm{HSV}$ and/or to the $\mathrm{YCrCb}$ systems (for the $\mathrm{Y}$ channel, $\mathrm{Y}=0.299 \mathrm{R}+0.587 \mathrm{G}+0.114 \mathrm{~B}$ )

8. Compute the texture features (SF, SGLDM, and GLDM at different scales) on the $\gamma$-corrected ROIs of step 7 for each of the channels of the color systems.

9. Classify ROIs into normal/abnormal using: a. SVM classifier, and/or b. PNN classifier.

10. Store raw video, ROIs, texture features, and classification results into hysteroscopy imaging database.

11. Perform visual expert analysis of the results (also supported via the web platform-taking into account the findings documented in the electronic patient record).

Reporting/Diagnosis (new)

12. Generate electronic patient record and hysteroscopy imaging $\mathrm{CAD}$ reports (including biopsy findings).

Fig. 2. Hysteroscopy imaging CAD protocol covering image calibration and color correction, video acquisition, texture analysis and classification, and diagnosis. This protocol was updated from previously published protocols by our group in [5], [7], and [26].

suspected endometrial lesions, and 2) 22 patients with normal endometrium. The mean age was 54.5 years (range $47-62$ ). The patients underwent a routine examination procedure covering family history, clinical examination and transvaginal ultrasound evaluation. In 16 (40\%) patients, endometrial lesions-like polyps and myomas were diagnosed and suspected to be the cause for abnormal bleedings. Diagnostic and operative hysteroscopy was performed under general anesthesia.

Initially, the expert surgeon performed a visual classification of suspect, normal endometrium regions. To verify the benign classification, we additionally performed three biopsies of the endometrium and endometrial curettage (blind scratch of the endometrium). When expert visual classification suggested endometrial pathology like polyps, myomas, erosion, or atrophy, biopsies were taken from the lesion as well as from the surrounding regions considered normal.

The videos were recorded and analyzed by the CAD system. From these videos, 416 ROIs of $64 \times 64$ pixels were cropped and classified into two categories based on the opinion of the 
physician: 1) for the normal case $(N=208)$ and 2$)$ for the abnormal case $(N=208)$. These cases were further verified based on the results of the histopathological examination. In order to investigate the accuracy of our classification approach, we also extracted an additional 100 ROIs using 50 ROIs extracted from ten normal cases and another 50 ROIs extracted from two abnormal cases.

\section{B. Video Recording}

Digital video images were acquired using the KARL STORZ Camera system [14], which included a luminance source and monitor. The camera was white balanced based on the manufacturer guidelines. The camera had an analog output signal (PAL 475 horizontal lines) that was digitized at $720 \times 576$ pixels using the VCE-PRO frame grabber [15] (24 bits color at 25 frames per second).

\section{Multiscale and Texture Feature Extraction}

We also investigated the use of different image scales as previously reported in [16]. Here, following low-pass filtering, each ROI was downsampled at rates that varied from $2 \times 2$ to $9 \times 9$.

A unique characteristic of our dataset comes from the need to characterize color texture content of the ROIs. We are thus led to consider texture features that are extracted over three different color spaces: RGB, YCrCb, and HSV. For each color space component, we consider the use of standard gray-scale features that have been widely applied for texture characterization. Furthermore, we also consider the combination of texture features over the entire color space. In other words, we consider the use of texture features extracted from all three components of each color space. A total number of 26 texture features were extracted from each color component. The list includes: 1) statistical features (SF) [17], 2) spatial gray-level dependence matrices (SGLDMs) [18], and 3) gray-level difference statistics (GLDS) [19]. The following SF texture features were computed: 1) mean, 2) variance, 3) median, 4) energy, 5) skewness, 6) kurtosis, 7) mode, and 8) entropy. The following SGLDM texture features were extracted:

1) angular second moment (ASM);

2) contrast;

3) correlation;

4) homogeneity;

5) variance;

6) entropy;

7) sum entropy;

8) sum average;

9) sum variance

10) difference entropy;

11) difference variance;

12) information measurement of correlation 1 ;

13) information measurement of correlation 2.

The following GLDS texture features were computed:

1) mean;

2) entropy;

3) contrast;
4) homogeneity;

5) energy.

\section{ROI Classification}

We investigated the performance of the system using support vector machines (SVMs) and probabilistic neural networks (PNNs). For both classifiers, we performed training and testing for differentiating between normal ROIs and abnormal ROIs.

The C-SVM network [20] was investigated using the Gaussian radial basis function (RBF) kernel and the linear kernel. Significantly better performance was obtained using the RBF kernel tuned based on the methodology proposed in [20]. More specifically, the values of $c=8$ and $\gamma=0.04$ were selected for prescribing the shape of the RBF kernel. These settings were fine tuned based on numerous runs for different feature sets using tenfold cross validation for 208 normal and 208 abnormal ROIs. We also consider the use of a PNN classifier that is based on the use of RBFs [21]. This classifier was investigated for several spread radii in order to identify the optimal value following a similar procedure as prescribed for the SVM case.

The leave-one-out method was used for validating all the classification models unless otherwise stated. The runs were done for each of the three color systems and for the Y channel. A total of $4 \times 415$ runs were carried out for each model, and the performance of the classifiers was evaluated on the remaining one subset.

\section{RESULTS}

Fig. 1 presents a screenshot of the proposed CAD system. An overview of the hysteroscopic imaging CAD system is demonstrated in Fig. 1(a). The system provides: patient information, video player, ROI classification into normal or abnormal, and texture features analysis. Fig. 1(b) presents a screenshot of the ROI capture mode (manual or semiautomatic) and the analysis results tab.

\section{A. Texture Feature Analysis}

To verify that the feature distributions did not follow a normal distribution, we used a Kolmogorov-Smirnov test at a significance level of $\alpha=0.05$. The test was applied after making the distribution of each feature to be zero mean and of unit variance for comparison to an $N(0,1)$ distribution. For all color spaces, we found that none of the extracted texture features followed a normal distribution. Thus, in testing for significant differences between texture features extracted from normal and abnormal ROIs, we adopt Wilcoxon test, which is nonparametric.

In Table I, we provide the results from statistical tests at different significant levels. At the most basic level (S1), we have statistical testing at a significance level of $\alpha=0.05$. This testing is commonly used for identifying individual texture feature differences [22]. Given the fact that we are extracting 26 features per color channel, to account for multiple comparison effects, we also performed testing at a significance level of $\alpha=0.05 / 26$ $(=0.002)$ using a Bonferroni correction as documented in [23] (S2 level). To also account for multiple effects within each 
TABLE I

Statistical ANALYsis of Selected TeXture Features For Normal (208) VERSUS ABNORMAL (208) ROIS OF THE ENDOMETRIUM EXTRACTED FROM 40 SUBJECTS FOR THE RGB, HSV, AND YCRCB COLOR SYSTEMS

\begin{tabular}{lccccccccc}
\hline \hline Channel & Y & Cr & Cb & H & S & V & R & G & B \\
\hline SF & & & & & & & & & \\
Mean & S2 & S1 & S1 & S3 & S1 & NS & NS & S2 & S1 \\
Variance & S3 & S3 & S3 & S3 & S3 & S3 & S3 & S3 & S3 \\
Median & S1 & S1 & S1 & S3 & S1 & NS & NS & S2 & S1 \\
Mode & NS & S1 & S1 & S3 & NS & NS & NS & S1 & NS \\
Energy & S2 & S3 & S3 & NS & S3 & S3 & S3 & S3 & S3 \\
Entropy & S3 & S3 & S3 & S3 & S3 & S3 & S3 & S3 & S3 \\
SGLDM & & & & & & & & & \\
ASM & S3 & S3 & S3 & S3 & S3 & S3 & S3 & S3 & S3 \\
Contrast & S3 & S3 & S1 & S3 & S1 & S3 & S3 & S3 & S2 \\
Correlation & S2 & S3 & S1 & NS & S3 & S3 & S3 & S3 & S3 \\
Variance & S3 & S3 & S3 & S3 & S3 & S3 & S3 & S3 & S3 \\
Homogeneity & S3 & S3 & S1 & S3 & S3 & S3 & S3 & S3 & S3 \\
Entropy & S3 & S3 & S3 & S3 & S3 & S3 & S3 & S3 & S3 \\
GLDS & & & & & & & & & \\
Homogeneity & S3 & S3 & S1 & S3 & S3 & S3 & S3 & S3 & S3 \\
Contrast & S3 & S3 & S1 & S3 & S1 & S3 & S3 & S3 & S2 \\
Energy & S3 & S3 & S1 & S3 & S1 & S3 & S3 & S3 & S3 \\
Entropy & S3 & S3 & S1 & S3 & S1 & S3 & S3 & S3 & S3 \\
Mean & S3 & S3 & S1 & S3 & S1 & S3 & S3 & S3 & S3 \\
\hline \hline
\end{tabular}

The Wilcoxon rank sum test was carried out with "S" and "NS" indicating significant and nonsignificant difference, respectively at: S1 level $\alpha=0.05$, S2 level $\alpha=0.05 / 26(=0.002)$, and S3 level $\alpha=0.05 /(26 * 3)(=0.0006)$ The table summarizes the highest significance level for which we have significant differences.

color space, we also performed testing at a significance level of $\alpha=0.05 / 26 * 3(=0.0006)$ (S3 level). In Table I, we report the highest level for which we detect significant differences between the normal and abnormal ROIs.

Fig. 3 shows the boxplots of the texture features SF median and SGLDM variance and contrast for the $\mathrm{YCrCb}$ channels for both the normal and abnormal endometrium ROIs. It is shown that for the $\mathrm{YCrCb}$ channels (see Fig. 3): 1) SF median for normal ROIs was higher compared to abnormal ROIs for the $\mathrm{Y}$ channel, whereas for the $\mathrm{Cr}$ and $\mathrm{Cb}$ channels, the abnormal ROIs gave slightly higher values; 2) SGLDM variance was higher for the abnormal ROIs for the YCrCb channels; and 3) SGLDM contrast was also higher for the abnormal ROIs for the $\mathrm{YCrCb}$ channels. These differences were also significantly different for the $\mathrm{YCrCb}$ channels at least for level S1 (see Table I). Additional features that demonstrated significant difference between normal and abnormal ROIs with the significance difference level given in parentheses were the following: SF energy (S2) and entropy (S3), SGLDM ASM (S3), homogeneity (S1), and entropy (S3); and GLDS homogeneity (S1), contrast (S1), energy (S1), entropy (S1), and mean (S1) (see Table I).

\section{B. CAD Classification Performance}

Classifier performance is summarized in Table II (scale = $1 \times 1$ ). For each classifier, we investigated the use of SF, SGLDM, and GLDS features sets extracted from Y, YCrCb, HSV, and RGB. From the results in Table II, it is clear that SVM performed better than PNN. In terms of color systems, $\mathrm{YCrCb}$ gave slightly better results, followed by HSV and RGB.
It's interesting to note that the classification performance of the $\mathrm{Y}$ channel only followed very closely the classification performance of the $\mathrm{YCrCb}$ system. For the SVM classifier, the best performance was achieved with the SF + GLDS features extracted from $\mathrm{YCrCb}$ system $(\% \mathrm{CC}=79 \%)$, followed by the $\mathrm{SF}$ + SGLDM + GLDS that gave $\% \mathrm{CC}=77 \%$ (for both using the original features sets).

Furthermore, multiscale analysis was carried out to derive the image feature scale that gives the best classification performance. However, we do not consider the selected scale to be part of the overall system. More specifically, the YCrCb SF + GLDS features sets (that gave the highest percentage of correct classifications score) were computed from the images at the following scales: $2 \times 2,3 \times 3,4 \times 4$, and $9 \times 9$. SVM and PNN classification models were then developed, and their performance is tabulated in Table III. It is shown that the best classification performance was achieved with the feature sets derived at the $1 \times 1$ image scale (i.e., $\% \mathrm{CC}=79 \%$ with the $\mathrm{SVM}$ classifier), whereas the feature sets derived at higher image scales $(2 \times 2$ up to $9 \times 9$ ) gave models with significantly lower $\% \mathrm{CC}$ (in the region of $60 \%$ with the SVM classifier).

In order to empirically establish the sufficiency of the number of training samples, we measured the classification performance as a function of the size of the training set. Here, we note that classification error is a nondecreasing function of the number of training samples (see [22] and [23]). Furthermore, empirically, as given in [23], increasing the number of training samples beyond what is needed will not result in significant increase in performance. As documented in Table IV, for our dataset, we obtained $79 \%$ for the original dataset of 416 ROIs that only increased to $81 \%$ with the addition of 100 ROIs from the second dataset (see description in Section II). Furthermore, classification performance was reduced to $76 \%$ for 316 ROIs, suggesting that our dataset size of 416 ROIs should not be reduced further. The ROC curves of these models are also given in Fig. 4. The area under the ROC curves was in the region of $0.865-0.870$ for the models with the highest performance (i.e., models 4 and 5 of Table IV).

Furthermore, the models documented in Table IV were also carried out using tenfold cross validation. The results were highly comparable but achieving a 1\%-2\% higher classification scores.

\section{DISCUSSION}

In this study, a CAD system was proposed based on color texture analysis for the analysis of hysteroscopy images of the endometrium. The system was evaluated on 258 normal and 258 abnormal ROIs (histopathologically verified) manually extracted by the gynecologist from 52 hysteroscopic imaging examinations (carried out on 52 subjects). It was shown that the $\mathrm{YCrCb}, \mathrm{SF}+\mathrm{GLDS}$ color texture features could correctly classify $81 \%$ of the cases (with a percentage sensitivity and specificity of $78 \%$ and $81 \%$, respectively) based on SVM modeling. Very similar but slightly lower classification performance was also obtained for the RGB and HSV color systems, as well as for the Y channel alone. Moreover, image features 


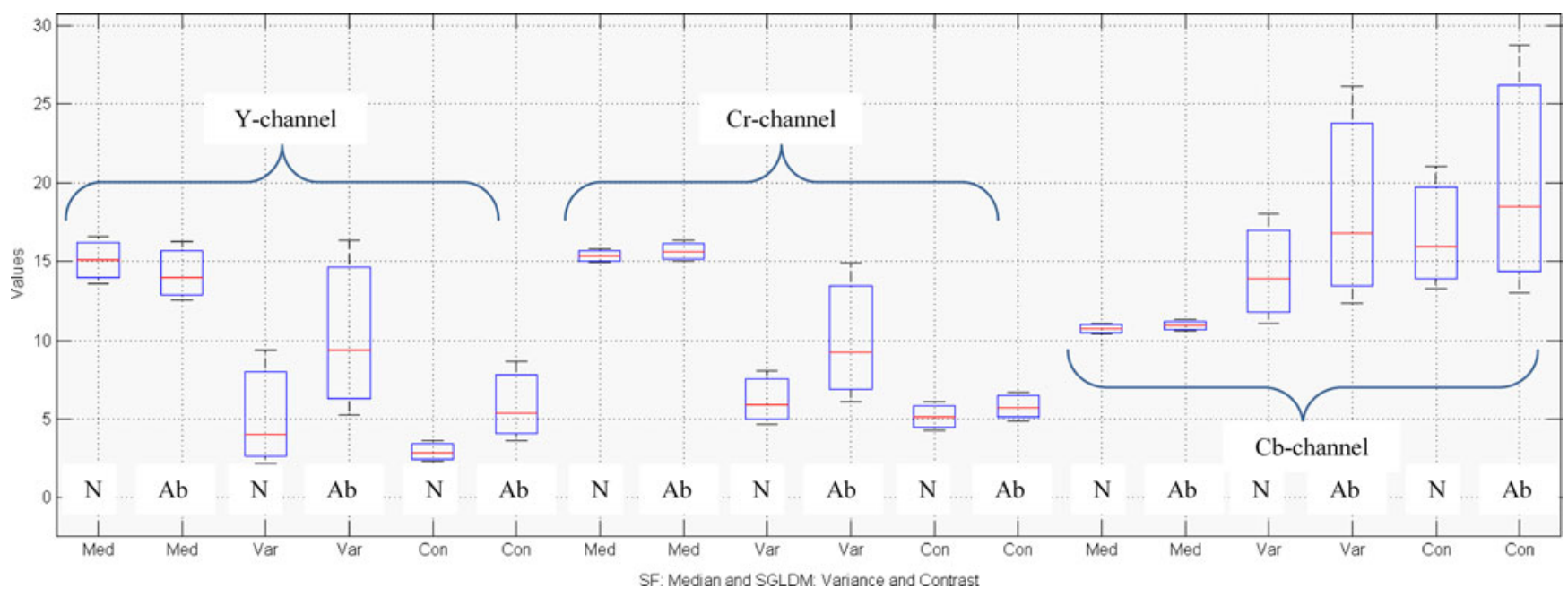

Fig. 3. Boxplot of SF median and SGLDM variance, contrast, for YCrCb channels, for normal (N) and abnormal (Ab) ROIs of the endometrium. The box shows the median, lower and upper quartiles and confidence interval around the median for each feature. The dashed lines are used to demonstrate the extent of each distribution to the outliers that are still considered to be part of the distribution. All these features have significant differences at least for $\alpha=0.05$ (S1 level) (see Table I for detailed statistical analysis).

TABLE II

Classification Performance of the CAD System BASEd on the SVM (PNN) MOdels to Classify Normal Versus AbNoRmal ROIS OF the ENDOMETRIUM BASED ON TEXTURE FEATURES FOR ORIGINAL TRANSFORMED FEATURES SETS

\begin{tabular}{|c|c|c|c|c|c|c|c|c|c|c|c|c|}
\hline $\begin{array}{l}\text { SVM (PNN) } \\
\text { Classifier }\end{array}$ & $\begin{array}{c}\% \mathrm{CC} \\
\mathrm{Y}\end{array}$ & RGB & HSV & $\mathrm{YCrCb}$ & $\mathrm{Y}$ & RGB & $\begin{array}{l}\% \text { SE } \\
\text { HSV }\end{array}$ & $\mathrm{YCrCb}$ & $\mathrm{Y}$ & RGB & $\begin{array}{l}\% \text { SP } \\
\text { HSV }\end{array}$ & $\mathrm{YCrCb}$ \\
\hline $\mathrm{SF}$ & $70(64)$ & $75(65)$ & $74(73)$ & $73(70)$ & $69(55)$ & $78(57)$ & $71(69)$ & $68(55)$ & $72(73)$ & $72(73)$ & $77(76)$ & $78(84)$ \\
\hline SGLDM & $76(67)$ & $72(67)$ & $73(72)$ & $74(69)$ & $75(62)$ & $67(62)$ & $70(67)$ & $76(50)$ & $77(72)$ & $77(72)$ & $76(77)$ & $71(88)$ \\
\hline GLDS & $72(67)$ & $69(63)$ & $73(73)$ & $75(70)$ & $65(51)$ & $59(44)$ & $68(68)$ & $75(53)$ & $79(82)$ & $79(82)$ & $78(77)$ & $75(86)$ \\
\hline $\mathrm{SF}+\mathrm{SGLDM}$ & $73(68)$ & $70(67)$ & $70(70)$ & $76(69)$ & $69(62)$ & $63(60)$ & $62(64)$ & $77(51)$ & $77(74)$ & $77(74)$ & $78(76)$ & $75(87)$ \\
\hline $\mathrm{SF}+\mathrm{GLDS}$ & $76(70)$ & $73(68)$ & $76(76)$ & $79(70)$ & $76(59)$ & $70(56)$ & $73(73)$ & $83(55)$ & $76(80)$ & $76(80)$ & $79(79)$ & $74(84)$ \\
\hline SGLDM+GLDS & $76(71)$ & $73(67)$ & $71(71)$ & $76(69)$ & $74(63)$ & $69(56)$ & $62(65)$ & $76(53)$ & $77(78)$ & $77(78)$ & $79(78)$ & $75(84)$ \\
\hline $\mathrm{SF}+\mathrm{SGLDM}+\mathrm{GLDS}$ & $74(72)$ & $73(68)$ & $76(69)$ & $77(69)$ & $66(65)$ & $64(58)$ & $73(60)$ & $79(53)$ & $82(78)$ & $82(78)$ & $79(78)$ & $74(85)$ \\
\hline
\end{tabular}

\%CC: percentage of correct classifications score; \%SE: percentage sensitivity; \%SP: percentage specificity.

TABLE III

Classification Performance of THE CAD System BASED on the SVM (PNN) MOdels to Classify NORMal Versus ABNORMAL ROIS OF THE ENDOMETRIUM BASED ON THE SF + GLDS FEATURE SETS FOR DIFFERENT SCALES FOR THE YCRCB SYSTEM

\begin{tabular}{|c|c|c|c|}
\hline \multirow[b]{2}{*}{ Scale } & \multicolumn{3}{|c|}{ YCrCb: SF + GLDS } \\
\hline & $\% \mathrm{CC}$ & $\% \mathrm{SE}$ & $\%$ SP \\
\hline $1 \times 1$ & $79(70)$ & $83(55)$ & $74(84)$ \\
\hline $2 \times 2$ & $61(57)$ & $50(30)$ & $71(85)$ \\
\hline $3 \times 3$ & $60(57)$ & $44(22)$ & $77(91)$ \\
\hline $4 \times 4$ & $59(56)$ & $43(18)$ & $74(94)$ \\
\hline $9 \times 9$ & $61(55)$ & $53(29)$ & $68(80)$ \\
\hline
\end{tabular}

analyzed at higher scales gave poorer classification performance. These findings were considered satisfactory by the gynecologist, supporting the differentiation between normal versus abnormal ROIs that could prove to be very helpful in the early detection of gynecological cancer.

The results of our study focused on early cancer detection are worse than the results reported by Vlachokosta et al. [8] for
TABLE IV

ClassificATION PERFormanCE OF THE CAD SySTEM BASED ON THE SVM MODELS TO CLASSIFY NORMAL VERSUS ABNORMAL ROIS OF THE ENDOMETRIUM BASED ON THE SF+GLDS FEATURE SETS FOR DIFFERENT TRAINING SETS FOR THE YCRCB SYSTEM

\begin{tabular}{lcccr}
\hline \hline & \multicolumn{3}{c}{ YCrCb: SF + GLDS } \\
\cline { 2 - 5 } No. of cases N/Ab & $\% \mathrm{CC}$ & $\%$ SE & $\%$ SP \\
\hline 1. & $78 / 78$ & 62 & 87 & 45 \\
2. & $128 / 128$ & 63 & 85 & 50 \\
3. & $158 / 158$ & 76 & 86 & 73 \\
4. & $208 / 208$ & 79 & 83 & 74 \\
5. & $258 / 258$ & 81 & 78 & 81 \\
\hline \hline
\end{tabular}

more advanced cancer cases. Vlachokosta et al. [8] reported a classification score of $91 \%$ that is higher than the $81 \%$ achieved by our method. However, the hysteroscopy cases investigated by Vlachokosta et al. [8] were clearly identified cases of malignancy that could easily be diagnosed by visualization alone. The formation of neoangiogenesis and vessels structure in the image data set that was investigated is considered to be a late 


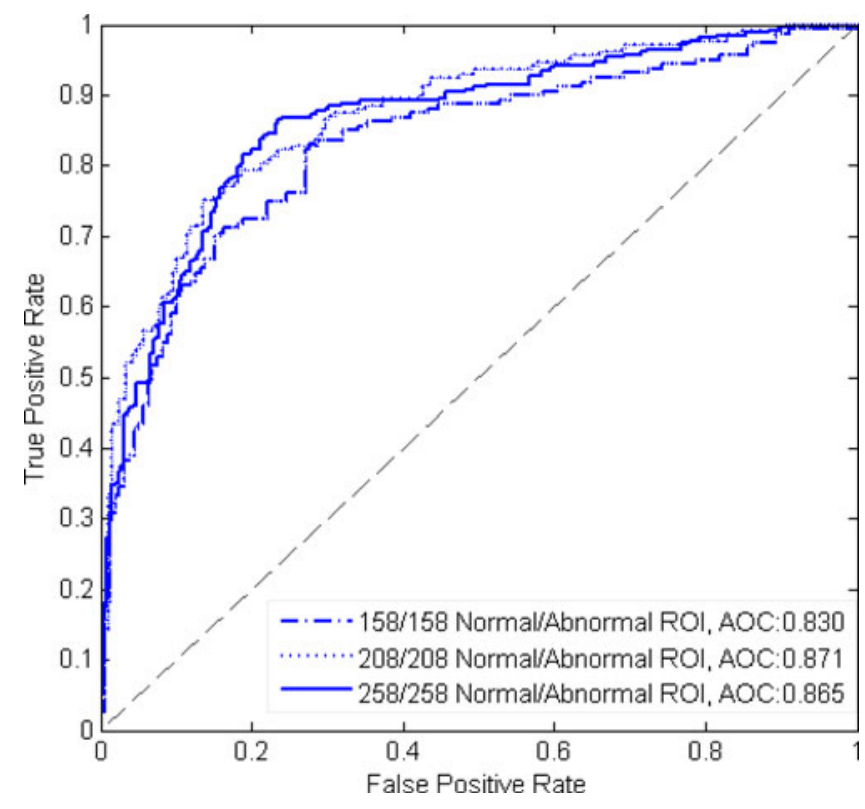

Fig. 4. ROC curves of models (3-5) tabulated in Table IV. The area under the ROC curve (AOC) is also given.

phenomenon of carcinogenesis. In contrast, in our study, the effort was targeted at investigating cases at the stage of occult cancer conditions at the level of initial cellular change to malignancy.

Endoscopy/laparoscopy/hysteroscopy video is widely used in medical practice during minimally invasive procedures to navigate in the human organ recognizing local anatomical landmarks. This task is mastered by the physician following extensive training and clinical practice and requires fine hand-eye coordination. Although in recent years, the endoscopic equipment manufacturers introduced better camera technologies (high-definition resolution, better three CCD sensors, and new optics and lenses) [14], limitations affecting the quantitative tissue analysis and computer-aided diagnosis still exist.

Thus, the proposed CAD system is limited by the limitations inherent in any endoscopy/laparoscopy/hysteroscopy examination. More specifically, these are the following: 1) the endoscope distance from the tissue (panoramic versus close-up view), 2) difference in viewing angles, 3) color correction, and 4) radial distortion.

It was shown in [5] that for 1) and 2), there was no significant difference in texture features, whereas for 3 ) it was shown that gamma-corrected images gave better image approximations to the original images. Limitation 4), i.e., radial distortion, is a serious problem that due to the small size of the endoscopic lenses used, nonlinear geometric deformations of the image are caused with points moving radially toward the center [24].

An innovative system to address this problem has recently been proposed by Melo et al. [25], featuring intrinsic real-time camera calibration, circular region segmentation, and inference of relative rotation between the lens probe and the camera head.
In conclusion, significant research work is still needed in several directions to enable the deployment of CAD hysteroscopic imaging systems in successful clinical practice. More specifically, there is a need to: 1) further enhance the proposed standardized CAD system with radial distortion correction, 2) investigate the performance of ROI image multiscale feature analysis like amplitude modulation-frequency modulation (AMFM) features that showed promising results in hysteroscopic imaging [26] as well as color wavelet features that were used successfully in endoscopic tumor detection [27], 3) incorporating navigation functionality for suspicious tissue localization and identification [28], and 4) evaluation on more subjects and in more clinics.

\section{REFERENCES}

[1] Cancer Fact and Figures. (2013). [Online]. Available: http://www. cancer.org/acs/groups/content/@epidemiologysurveilance/documents/ document/acspc-036845.pdf

[2] J. A. Fayez and M. F. Vogel, "Comparison of different treatment methods of endometriomas by laparoscopy," Obstet. Gynecol., vol. 78, pp. 660-665, 1991.

[3] J. Ogden, M. Heinrich, C. Potter, A. Kent, and S. Jones, "The impact of viewing a hysteroscopy on a screen on the patient's experience: A randomised trial," BJOG: Int. J. Obstetrics Gynaecol., vol. 116, pp. 286-293, 2009.

[4] R. Wenzl, R. Lehner, U. Vry, N. Pateisky, P. Sevelda, and P. Husslein, "Three-dimensional video-hysteroscopy: Clinical use in gynaecological laparoscopy," Lancet, vol. 344, pp. 1621-1622, 1994.

[5] M. S. Neophytou, C. S. Pattichis, M. S. Pattichis, V. Tanos, E. C. Kyriacou, and D. Koutsouris. (2007). A standardised protocol for texture feature analysis of endoscopic images in gynaecological cancer. Biomed. Eng. OnLine [Online]. 6(44). Available: http://www.biomedical-engineeringonline.com/content/6/1/44

[6] I. P. Constantinou, C. A. Koumourou, M. S. Neofytou, V. Tanos, C. S. Pattichis, and E. C. Kyriakou, "An integrated CAD system facilitating the endometrial cancer diagnosis," in Proc. 9th Int. Conf. Inf. Technol. Appl. Biomed., Larnaca, Cyprus, Nov. 5-7, 2009, pp. 1-5.

[7] M. S. Neofytou, V. Tanos, M. S. Pattichis, C. S. Pattichis, E. C. Kyriacou, and S. Pavlopoulos, "Color based texture-Classification of hysteroscopy images of the endometrium," in Proc. 29th Annu. Int. Conf. IEEE Eng. Med. Biol. Soc., Lyon, France, Aug. 23-27, 2007, pp. 864-867.

[8] A A. Vlachokosta, P. A. Asvestas, G. K. Matsopoulos, A. Kondi-Pafiti, and N. Vlachos, "Classification of histological images of the endometrium using texture features," Med. Biol. Eng. Comput., vol. 51, pp. 859-867, 2013.

[9] S. Sheraizin and V. Sheraizin, "Endoscopy imaging intelligent contrast improvement," in Proc. 27th Аnnu. Int. Conf. IEEE Eng. Med. Biol. Soc., Shanghai, China, Sep. 1-4, 2005, pp. 6551-6554.

[10] J. Scharcanski, W. Gaviao, S. Cunha, and F. Joao, "Diagnostic hysteroscopy video summarization and browsing," in Proc. 27th Annu. Int Conf. IEEE Eng. Med. Biol. Soc., Shanghai, China, Sep. 1-4, 2005, pp. $5680-5683$.

[11] W. Gaviao and J. Scharcanski, "Content-based diagnostic hysteroscopy summaries for video browsing," in Proc. 18th Brazilian Symp. SIBGRAPH, Comput. Graph. Image Process., vol. 2005, 2005, pp. $21-28$.

[12] Y. Peng, R. Jia, Y. Wang, and M. Zhang, "A virtual endoscopy system for virtual medicine," Comput. Animation Virtual Worlds, vol. 22, pp. 277-284, 2011.

[13] A. C. Bovik (Ed.), The Essential Guide to Image Processing. New York, NY, USA: Academic Press, 2009.

[14] (2013, Dec.). Cameras, light sources and documentation [Online] Available: https://www.karlstorz.com/cps/rde/xchg/SID-2CDAEDF1FAFACD43/karlstorz-en/hs.xsl/74.htm

[15] (2013, Dec.). VCE-PRO is a PCMCIA Cardbus video capture card [Online]. Available: http://www.imperx.com/frame-grabbers/vce-pro

[16] P. P. Vaidyanathan, Multirate Systems and Filter Banks. Englewood, NJ, USA: Prentice Hall, 1993. 
[17] C. M. Wu, Y. C. Chen, and K. S. Hsieh, "Texture features for classification of ultrasonic liver images," IEEE Trans. Med. Imaging, vol. 11, no. 2, pp. 141-152, Jun. 1992.

[18] R. M. Haralick, K. Shanmugam, and I. Dinstein, "Texture features for image classification," IEEE Trans. Syst. Man Cybern., vol. SMC-3, no. 6, pp. 610-621, Nov. 1973.

[19] J. S. Wenska, C. R. Dryer, and A. Rosenfeld, "A comparative study of texture measures for terrain classification," IEEE Trans. Syst. Man Cybern., vol. SMC-6, no. 4, pp. 269-285, Apr. 1976.

[20] C.-C. Chang and C.-J. Lin. (2011). LIBSVM: A library for support vector machines. ACM Trans. Intell. Syst. Technol. [Online], 2, pp. 27:1-27:27. Available: http://www.csie.ntu.edu.tw/ cjlin/libsvm.

[21] D. F. Specht, "Probabilistic neural networks," INNS Neural Netw., vol. 3, no. 1, pp. 109-118, 1990.

[22] T. Hastie, R. Tibshirani, and J. Friedman, The Elements of Statistical Learning: Data Mining, Inference, and Prediction, 2nd ed. Berlin, Germany: Springer, 2009.

[23] S. Theodoridis and K. Koutroumbas, Pattern Recognition, 4th ed. Amsterdam, The Netherlands: Elsevier, 2008.

[24] J. L. Fleiss, The Design and Analysis of Clinical Experiments. New York, NY, USA: Wiley Classics Library, 1999.
[25] R. Melo, J. Barreto, and G. Falcao, "A new solution for camera calibration and real-time image distortion correction in medical endoscopy-Initial technical evaluation," IEEE Trans. Biomed. Eng., vol. 59, no. 3, pp. 634 644, Mar. 2012.

[26] I. P. Constantinou, M. S. Neofytou, V. Tanos, M. S. Pattichis, and C. S. Pattichis, "An adaptive multiscale AM-FM texture analysis system with application to hysteroscopy imaging," in Proc. 12th IEEE Int. Conf. Bioinform. Bioeng., Larnaca, Cyprus, Nov. 11-13, 2012, pp. 744-747.

[27] S. A. Karkanis, D. K. Iakovidis, D. E. Maroulis, A. D. Karras, and M. Tzivras, "Computer-aided tumor detection in endoscopic video using color wavelet features," IEEE Trans. Inf. Technol. Biomed., vol. 7, no. 3, pp. 141-152, Sep. 2003.

[28] I. Herakleous, I. P. Constantinou, E. Michael, M. S. Neofytou, C. S. Pattichis, and V. Tanos, "A simplified 2D real time navigation system for hysteroscopy imaging," in Proc. Int. Conf. Comput. Syst. Technol. CompSysTech'11, Vienna, Austria, Apr. 2011, pp. 347-352.

Authors' photographs and biographies not available at the time of publication. 